\title{
ARGUMENTAC̣ÃO NO ENSINO DE CIÊNCIAS: CONTEXTO BRASILEIRO
}

\author{
Luciana Passos Sá* \\ Salete Linhares Queiroz *
}

RESUMO: No presente artigo discutimos a produção acadêmica brasileira sobre a argumentação no ensino de Ciências. Para tanto, foram investigados trabalhos apresentados nos Encontros Nacionais de Pesquisa em Educação em Ciências (ENPECs) e em revistas nacionais das áreas de Educação e Educação em Ciências. Os trabalhos foram analisados com relação aos seguintes aspectos: ano de publicação, região geográfica e Instituição de Ensino Superior (IES) de origem, nível de escolaridade ao qual se destina e foco temático.

Palavras-chave: Argumentação; Revisão Bibliográfica; Ensino de Ciências.

\section{ARGUMENTATION IN SCIENCE EDUCATION: BRAZILIAN CONTEXT}

ABSTRACT: In this very article, the Brazilian academic production on argumentation in Science Education is discussed. Therefore, studies presented at Encontros Nacionais de Pesquisa em Educação em Ciências *Doutora em Ciências pela
Universidade Federal de São
Carlos (UFSCar). Professora
do Departamento de
Ciências Exatas
e Tecnológicas da
Universidade Estadual de
Santa Cruz (UESC)
E-mail:
Iucianapsa@gmail.com
* *Doutora em Química pela
Universidade Estadual
Paulista (UNESP/Araraquara).
Professora Livre-Docente do
Instituto de Química de São
Carlos da Universidade de
São Paulo (USP).
E-mail:
salete@iqsc.usp.br (ENPECs - Brazilian Meetings for Research in Science Education) and published in Brazilian journals on Education and Science Education were investigated. This literature was reviewed comprising the following aspects: year of presentation, Brazilian geographic region and institution of production, school level and thematic focus of the study.

Keywords: Argumentation; Literature Review; Science Education. 


\section{INTRODUÇÃO}

Questões concernentes à argumentação se apresentam como temáticas emergentes nas pesquisas que tratam do papel da linguagem no processo de ensino-aprendizagem. Segundo Nascimento e Vieira (2008), estudos a respeito se iniciaram na década de 1950, a partir das obras de Perelman e OlbrechtsTyteca e de Toulmin, sendo frutos do desenvolvimento das abordagens críticas e dialogais sobre o pensamento e a linguagem. Nessa perspectiva, discussões sobre questões relacionadas à argumentação no ensino de Ciências têm sido reportadas com frequência na literatura (SADLER, 2006; KOLSTO, 2006; LEE et al, 2009). No contexto internacional merece destaque a publicação do livro Argumentation in Science Education (ERDURAN \& JIMENEZ ALEIXANDRE, 2008), que reúne trabalhos de renomados especialistas no tema. O livro apresenta pesquisas que combinam perspectivas teóricas e empíricas relevantes para o ensino de Ciências. Também é digna de nota a publicação do número especial Argumentación en el Salón de Clase, na seção De aniversario da revista mexicana Educación Quimica, em 2009. A seção conta com seis contribuições de pesquisas provenientes de diferentes países, duas delas desenvolvidas no Brasil: O Espaço para a Argumentação no Ensino Superior de Química, de Queiroz \& Sá (2009), do Grupo de Pesquisa em Ensino de Química do Instituto de Química de São Carlos, Universidade de São Paulo (IQSC/USP); e Argumentación en Textos de una Revista de Educación de Profesores. Un Ejercicio de Análisis Basado en Conceptos Bakbtinianos de Gênero y Lenguaje Social, de Martins (2009), do Laboratório de Linguagens e Mediações, da Universidade Federal do Rio de Janeiro (UFRJ). Tais pesquisas sinalizam que os modos dialógicos de interação são elementos essenciais do processo de ensino-aprendizagem e que os professores necessitam estar preparados para fomentá-los na sala de aula.

No contexto nacional merece destaque a publicação do livro Argumentação e ensino de Ciências, organizado por Nascimento e Plantin (2009), que discute a natureza do discurso científico, não somente argumentativo.

Tendo em vista a crescente atenção dispensada ao assunto em questão, no presente manuscrito apresentamos o estudo realizado sobre o estado da arte das pesquisas que tratam da argumentação no ensino de Ciências no âmbito nacional, no qual buscamos identificar os focos temáticos privilegiados por diferentes autores oriundos de diferentes instituições de ensino distribuídas nas distintas regiões brasileiras. Buscamos, ainda, verificar as principais tendências no que diz respeito ao nível de escolaridade privilegiado nas pesquisas e área de ensino envolvida. 


\section{METODOLOGIA}

Neste manuscrito foram analisados trabalhos relacionados à argumentação na Educação em Ciências apresentados no evento nacional mais abrangente da área de Educação em Ciências, o Encontro Nacional de Pesquisa em Educação em Ciências (ENPEC), no período de 1997 a 2009, e aqueles publicados em revistas brasileiras que constam na área de avaliação do Programa QUALIS da Coordenação de Aperfeiçoamento de Pessoal de Nível Superior (CAPES). O Programa QUALIS consiste em um conjunto de procedimentos utilizados pela CAPES para estratificação da qualidade da produção intelectual dos programas de Pós-Graduação. Dessa maneira, consultamos periódicos da área 38 (Educação) que se relacionam ao ensino de Ciências; e da área 46 (Ensino de Ciências e Matemática). O levantamento foi realizado desde o ano inicial de publicação de cada uma das revistas analisadas até a última edição disponível, com exceção apenas da Revista Química Nova, cujo levantamento foi realizado a partir do ano de 1994, uma vez que, diferentemente das demais, tem a sua origem ainda nos anos 70 .

Realizamos a busca primeiramente pela verificação de menções à argumentação ou termos similares nos títulos dos trabalhos e palavras-chave. Em seguida, a leitura dos trabalhos, na íntegra, foi realizada. As seguintes etapas foram seguidas: definição dos aspectos a serem considerados na classificação e descrição dos trabalhos em estudo; organização dos dados bibliográficos e de informações complementares em uma ficha de classificação; leitura e classificação dos documentos com relação aos aspectos definidos anteriormente; organização dos resultados da classificação dos documentos com relação aos vários aspectos analisados em forma de tabelas e gráficos e análise dos resultados e elaboração das principais tendências verificadas nos documentos. Assim, buscamos apresentar um panorama a respeito da produção acadêmica sobre o tema argumentação, com ênfase nos seguintes aspectos: (a) A produção e sua distribuição no tempo; (b) A produção e sua distribuição de acordo com a área de pesquisa; (c) A produção e sua distribuição de acordo com as regiões brasileiras; (d) A produção e sua distribuição de acordo com as instituições acadêmicas; (e) A produção e sua distribuição de acordo com os níveis de escolaridade e (f) A produção e sua distribuição de acordo com o foco temático.

\section{RESULTADOS E DISCUSSÃO}

Conforme ilustra a Tabela 1 , localizamos nas revistas doze trabalhos publicados por grupos de pesquisa nacionais que abordam questões relacionadas à argumentação no ensino de Ciências. Vale ressaltar que localizamos três trabalhos provenientes do exterior que foram desconsiderados na análise. As revistas Química Nova na Escola, Revista Brasileira de Ensino de Química, Ciência \& Ensino, 
Ciência \& Cognição, Revista Brasileira de Ensino de Física, A Física na Escola e Revista Brasileira de Ensino de Bioquímica e Biologia Molecular também foram analisadas, porém nenhum trabalho foi localizado.

Tabela 1 - Periódicos analisados, quantidade de trabalhos localizados em cada um deles e os respectivos períodos em que o levantamento foi realizado.

\begin{tabular}{c|c|c} 
Periódicos & Período & Número de trabalhos \\
\hline Química Nova & 1994 a 2009 & $\mathbf{1}$ \\
\hline Revista Brasileira de Pesquisa em Educação em Ciências (RBPEC) & 2001 a 2009 & $\mathbf{2}$ \\
\hline Ciência \& Educação & 1998 a 2009 & $\mathbf{2}$ \\
\hline Investigações em Ensino de Ciências & 1996 a 2009 & $\mathbf{3}$ \\
\hline Ensaio: Pesquisa em Educação em Ciências & 1999 a 2009 & $\mathbf{2}$ \\
\hline Caderno Brasileiro de Ensino de Física & 1993 a 2009 & $\mathbf{2}$
\end{tabular}

A Tabela 2 apresenta a lista de trabalhos publicados nas revistas mencionadas, em ordem cronológica.

Tabela 2 - Artigos sobre 0 tema argumentação publicados em revistas brasileiras.

\begin{tabular}{|c|c|}
\hline Autores e Título & Publicação e Ano \\
\hline $\begin{array}{l}1 \text { - CAPECCHI, M. C. V. M.; CARVALHO, A. M. P. Argumentação em uma aula de conhecimento } \\
\text { físico com crianças na faixa de oito a dez anos. }\end{array}$ & $\begin{array}{l}\text { Investigações em Ensino de Ciências, } \\
\text { v. } 5, \text { n. 3, p. 171-189, } 2000 .\end{array}$ \\
\hline $\begin{array}{l}2 \text { - SANTOS, W. P.; MORTIMER, E. F.; SCOTT, P. H. A argumentação em discussões sócio-cien- } \\
\text { tíficas: reflexões a partir de um estudo de caso. }\end{array}$ & $\begin{array}{l}\text { Revista Brasileira de Pesquisa em } \\
\text { Educação em Ciências, v. 1, n. 1, } \\
\text { p. 140-152, } 2001 .\end{array}$ \\
\hline $\begin{array}{l}3 \text { - CAPECCHI, M. C. V. M.; CARVALHO, A. M. P.; SILVA, V. Relações entre o discurso do profes- } \\
\text { sor e a argumentação dos alunos em uma aula de Física. }\end{array}$ & $\begin{array}{l}\text { Ensaio: Pesquisa em Educação em } \\
\text { Ciências, v. 2, n. 2, p. 189-208, } 2002 .\end{array}$ \\
\hline $\begin{array}{l}4 \text { - VILLANI, C. E. P.; NASCIMENTO, S. S. A argumentação e o Ensino de Ciências: uma atividade } \\
\text { experimental no laboratório didático de Física do Ensino Médio. }\end{array}$ & $\begin{array}{l}\text { Investigações em Ensino de Ciências, } \\
\text { v. 8, n. 3, p. 1-15, } 2003 .\end{array}$ \\
\hline 5 - SÁ, L. P.; QUEIROZ, S. L. Promovendo a argumentação no Ensino Superior de Química. & $\begin{array}{l}\text { Química Nova, } \\
\text { v. 30, n. 8, p. 2035-2042, } 2007 .\end{array}$ \\
\hline $\begin{array}{l}6 \text { - ASSIS, A.; TEIXEIRA, O. P. B. Dinâmica discursiva e o ensino de Física: análise de um episó- } \\
\text { dio de ensino envolvendo o uso de um texto alternativo. }\end{array}$ & $\begin{array}{l}\text { Ensaio: Pesquisa em Educação em } \\
\text { Ciências, v. 9, n. 2, p. 177-190, } 2007 .\end{array}$ \\
\hline $\begin{array}{l}\text { - VIEIRA, R. D.; NASCIMENTO, S. S. A argumentação no discurso de um professor e seus estu- } \\
\text { dantes sobre um tópico de mecânica newtoniana. }\end{array}$ & $\begin{array}{l}\text { Caderno Brasileiro de Ensino de Física, } \\
\text { v. } 24, \text { n. 2, p. 174-193, } 2007 .\end{array}$ \\
\hline $\begin{array}{l}8 \text { - NASCIMENTO, S. S.; VIEIRA, R. D. Contribuições e limites do padrão de argumento de Toulmin } \\
\text { aplicado em situações argumentativas de sala de aula de Ciências. }\end{array}$ & $\begin{array}{c}\text { Revista Brasileira de Pesquisa em } \\
\text { Educação em Ciências, v. 8, n.2, } 2008 .\end{array}$ \\
\hline $\begin{array}{l}9 \text { - CIRINO, M. M.; SOUZA, A. R. O discurso de alunos do Ensino Médio a respeito da "camada de } \\
\text { ozônio". }\end{array}$ & $\begin{array}{c}\text { Ciência \& Educação, } \\
\text { v. } 14, \text { n.1, p. } 115-134,2008 .\end{array}$ \\
\hline $\begin{array}{l}10 \text { - NASCIMENTO, S. S.; PLANTIN, C.; VIEIRA, R. D. A validação de argumentos em sala de aula: } \\
\text { um exemplo a partir da formação inicial de professores de Física. }\end{array}$ & $\begin{array}{l}\text { Investigações em Ensino de Ciências, } \\
\text { v. 13, n. 2, p. 169-185, } 2008 .\end{array}$ \\
\hline $\begin{array}{l}11 \text { - ASSIS, A.; TEIXEIRA, O. P. B. Argumentações discentes e docente envolvendo aspectos } \\
\text { ambientais em sala de aula: uma análise. }\end{array}$ & $\begin{array}{l}\text { Ciência \& Educação, } \\
\text { v. } 15, \text { n.1, p. } 47-60,2009 .\end{array}$ \\
\hline $\begin{array}{l}12 \text { - VIEIRA, R. D.; NASCIMENTO, S. S. Uma proposta de critérios marcadores para identificação } \\
\text { de situações argumentativas em salas de aulas de Ciências. }\end{array}$ & $\begin{array}{l}\text { Caderno Brasileiro de Ensino de Física, } \\
\text { v. } 26 \text {, n. 1, p. 81-102, } 2009 .\end{array}$ \\
\hline
\end{tabular}

A Tabela 3 ilustra os títulos e os autores dos trabalhos apresentados em todas as edições dos ENPECs, também em ordem cronológica. 
Tabela 3 - Trabalhos completos sobre 0 tema argumentação apresentados nos ENPECs.

ENPEC

III ENPEC - Atibaia, 2001

13 - A argumentação em discussões sócio-científicas: reflexões a partir de um estudo de caso.

14 - Argumentação: análises a partir de um princípio de pesquisa vivenciado em sala de aula.

$\mathbf{1 5}-0$ conhecimento físico e a linguagem.

IV ENPEC - Bauru, 2003

16 - Atividades experimentais de demonstração e o discurso do professor no ensino de Física.

17 - Argumentação situada: trazendo a argumentação científica para o contexto da Ciência escolar. V ENPEC - Bauru, 2005

18 - Práticas discursivas e 0 ensino-aprendizagem do professor de Ciências: tecendo relações entre argumentação e objetivos pedagógicos na formação inicial.

19 - Análise de um episódio de ensino envolvendo 0 uso de um texto paradidático em aulas de Física em uma sala de educação de jovens e adultos.

20 - Atividades promotoras de argumentação nas séries iniciais: 0 que fazem os professores? VI ENPEC - Florianópolis, 2007

21 - Fazeres pedagógicos e pesquisa sobre argumentação no Ensino de Ciências.

22 - Procedimentos discursivos didáticos de um formador em situações argumentativas na formação inicial de professores de Física.

23 - Argumentação e abordagem contextual: ensinando a síntese newtoniana.

24 - Argumentação no Ensino de Ciências: resultados de pesquisas.

25 - Argumentação no Ensino Superior de Química: reflexões a partir das interações estabelecidas na sala de aula.

26 - Análise de uma atividade experimental que desenvolva a argumentação dos alunos.

27 - Casos investigativos no ensino do tópico "corrosão". VII ENPEC - Florianópolis, 2009

28 - A argumentação em uma atividade experimental investigativa no Ensino Médio de Química. 29 - Proposição de um instrumento para avaliação de habilidades argumentativas - parte I fundamentos teóricos.

30 - Proposição de um instrumento para avaliação de habilidades argumentativas - parte II validação.

31 - A sala de aula, uma arena argumentativa: o debate entre alunos como veículo da construção coletiva de conhecimentos.

32 - Argumentação em salas de aula de Biologia sobre a teoria sintética da evolução.

33 - 0 discurso em sala de aula gerenciado por um professor estagiário: um instrumento para a formação de professores de Física.

34 - Epistemologia, argumentação e explicação na sequência didática de um livro de Química.

35 - Focos de pesquisa em argumentação no Ensino de Ciências: analisando o referencial teórico.

36 - Análise da argumentação em uma atividade investigativa de Biologia no Ensino Médio.

37 - A argumentação na produção escrita de professores de Ciências e 0 ensino da Genética.

38 - Temas sócio-científicos: análise dos processos argumentativos num contexto escolar.

39 - Os professores de Ciências devem ensinar os alunos a argumentar?

40 - Uma análise sobre as características de argumentos de alunos do Ensino Médio sobre temática sóciocientífica.

41 - Argumentação científica em um filme infanto-juvenil e na escrita dos alunos: uma relação possível?

42 - Modelo de argumentação como ferramenta para análise da qualidade da escrita científica de alunos de graduação em Química.

43 - A inquirição como instrumento para promover o raciocínio e argumentação em sala de aula.
Autores

Santos, W. P.; Mortimer, E. F.; Scott, P. H.

Lima, V. M. R.; Moraes, R.; Ramos, M. G. Oliveira, C. M. A.; Carvalho, A. M. P.

Monteiro, I. C. C; Monteiro, M. A. A; Gaspar, A. Munford, D.; Zembal-Saul, C.

Munford, D.; Lopes, M. G.; Tavares, F. P.; Vieira, R. D.

Assis, A.; Teixeira, 0. P. B.

Teixeira, F. M.

Teixeira, F. M.

Vieira, R. D.; Nascimento, S. S.

Teixeira, E. S.; Silva Neto, C. P.; Freire Jr.; 0. Winch, P. G.; Terrazzan, E. A.

Sá, L. P. ; Queiroz, S. L.

Biosoto, J. E.; Carvalho, A. M. P. C.

Velloso, A. M. S.; Sá, L. P.; Queiroz, S. L.

Suart, R.C; Marcondes, M. E. R. Mendonça, P. C. C.; Justi, R.

Mendonça, P. C. C.; Correa, H. L. S.; Justi, R.

Charret, H. C.; Conceição, W. M. N.

Tavares, M. L.; Mortimer, E.F.; El-Hani, C. N. Vieira, R. D.; Nascimento, S. S.

Silva, G. J.; Martins, C. M. Bozzo, M. V.; Motokane, M. T.

Silva, R. P. O.; Chernicharo, P. S. L.;

Tonidandel, S. M. R.; Trivelato, S. L. F. Valle, M. G.; Motokane, M. T. Bortoletto, A.; Carvalho, W. L. P.

Sá, L. P.; Ferreira, J. Q.; Queiroz, S. L. Pereira, R. G.; Trivelato, S. L. F.

Dell Asem, E. C. A.; Trivelato, S. L. F.

Oliveira, J. R. S.; Batista, A. A.; Queiroz, S. L. Manzano, M. E.; Faht, E. C.

\section{A produção e sua distribuição no tempo}

Com base no levantamento bibliográfico constatamos que, a partir do ano de 2007, cresceu o número de trabalhos publicados sobre questões ligadas à argumentação. Na Figura 1 apresentamos a frequência de trabalhos localizados nas revistas, de acordo com os períodos especificados na Tabela 1, e aqueles apresentados nos ENPECs. 
No que diz respeito às revistas analisadas, o primeiro trabalho localizado sobre a temática foi publicado no ano de 2000 e até 2003 a frequência de trabalhos por ano permanece inalterada, apenas um a cada ano. De 2004 a 2006, nenhum trabalho foi publicado no Brasil sobre a argumentação e somente em 2007 tornam a surgir pesquisas sobre a temática. Nesse ano, três trabalhos foram localizados, mesmo número verificado em 2008. No que diz respeito a 2009, o último número de algumas das revistas analisadas ainda não se encontra disponível para consulta e, por essa razão, alguns trabalhos do referido ano podem não constar na análise.

Figura 1 - Quantidade de trabalhos localizados nas revistas e apresentados nos ENPECs.

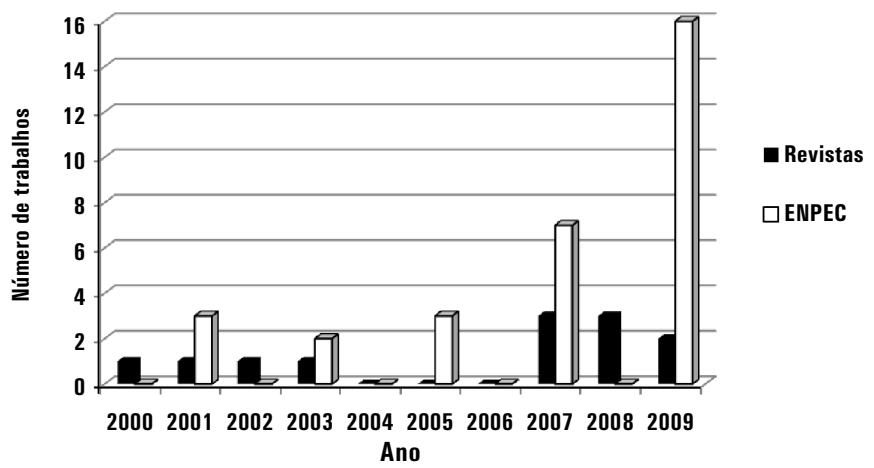

Como é possível observar na Figura 1, é notório o crescimento do número de trabalhos apresentados nos ENPECs, no decorrer dos anos, a respeito do tema em foco. Nos dois primeiros Encontros, ocorridos em 1997 e 1999, não identificamos a existência de nenhum trabalho sobre a temática e, somente em 2001, são apresentados os primeiros estudos. Vale ressaltar que um desses trabalhos, o desenvolvido por Santos et al (2001), aparece na Tabela 2 e 3, isso porque foi apresentado no III ENPEC e publicado na Revista Brasileira de Pesquisa em Educação em Ciências, no mesmo ano. A partir de 2005, cresce significativamente o número de trabalhos apresentados no evento, com um salto acentuado no último ENPEC, realizado em 2009, que contou com a apresentação de dezesseis trabalhos. Vale destacar que o ENPEC é um evento que ocorre a cada dois anos, por essa razão na Figura 1 nenhum trabalho é verificado nos anos pares.

Como é possível observar, poucos trabalhos foram localizados nas revistas da área, porém foi significativo o número de trabalhos apresentados no último ENPEC. Esses dados sugerem que o interesse pelo tema se acentuou nos últimos anos.

Na Tabela 4 é apresentada a classificação de cada um dos referidos trabalhos de acordo com a área de enfoque, a região brasileira e a instituição de origem, aspectos a serem discutidos nos tópicos seguintes. Os números atribuídos 
aos trabalhos correspondem à numeração dos trabalhos nas Tabelas 2 e 3. Doravante, os trabalhos serão referenciados por seus respectivos números.

Tabela 4 - Classificação dos trabalhos de acordo com a área de enfoque, região brasileira e instituição de origem.

\begin{tabular}{|c|c|c|c|c|}
\hline$N^{0}$ doTrabalho & Tabela & Área & Região Brasileira & Instituição \\
\hline 1 & 2 & Física & Sudeste & USP \\
\hline 2 & 2 & Química & Centro-oeste/Sudeste & UnB/UFMG/Univ. Leeds \\
\hline 3 & 2 & Física & Sudeste & USP \\
\hline 4 & 2 & Física & Sudeste & UFMG \\
\hline 5 & 2 & Química & Sudeste & UFSCar/USP \\
\hline 6 & 2 & Física & Sudeste & UNESP \\
\hline 7 & 2 & Física & Sudeste & UFMG \\
\hline 8 & 2 & Física & Sudeste & UFMG \\
\hline 9 & 2 & Química & Sudeste & UNESP \\
\hline 10 & 2 & Física & Sudeste & UFMG \\
\hline 11 & 2 & Física & Sudeste & UNESP \\
\hline 12 & 2 & Física & Sudeste & UFMG \\
\hline 13 & 3 & Química & Centro-oeste/Sudeste & UnB/UFMG/Univ. Leeds \\
\hline 14 & 3 & Química & Sul & PUC/RS \\
\hline 15 & 3 & Física & Sudeste & USP \\
\hline 16 & 3 & Física & Sudeste & UNESP \\
\hline 17 & 3 & Ciências & Sudeste & UFMG/Univ. Pensilvânia \\
\hline 18 & 3 & Ciências & Sudeste & UFMG \\
\hline 19 & 3 & Física & Sudeste & UNESP \\
\hline 20 & 3 & Ciências & Nordeste & UFPE \\
\hline 21 & 3 & Ciências & Nordeste & UFPE \\
\hline 22 & 3 & Física & Sudeste & UFMG \\
\hline 23 & 3 & Física & Nordeste & UEFS/UFBA \\
\hline 24 & 3 & Ciências & Sul & UFSM \\
\hline 25 & 3 & Química & Sudeste & UFSCar/USP \\
\hline 26 & 3 & Física & Sudeste & USP \\
\hline 27 & 3 & Química & Sudeste & UFSCar/USP \\
\hline 28 & 3 & Química & Sudeste & USP \\
\hline 29 & 3 & Ciências & Sudeste & UFMG \\
\hline 30 & 3 & Ciências & Sudeste & UFMG \\
\hline 31 & 3 & Física & Sudeste & Escola SESC/RJ \\
\hline 32 & 3 & Biologia & Sudeste/Nordeste & UFMG/UFBA \\
\hline 33 & 3 & Física & Sudeste & UFMG \\
\hline 34 & 3 & Química & Sudeste & UFMG \\
\hline 35 & 33 & Ciências & Sudeste & USP \\
\hline 36 & 3 & Biologia & Sudeste & USP \\
\hline 37 & 3 & Ciências & Sudeste & USP \\
\hline 38 & 3 & Ciências & Sudeste & UNESP \\
\hline 39 & 3 & Química & Sudeste & UFSCar/USP \\
\hline 40 & 3 & Ciências & Sudeste & USP \\
\hline 41 & 3 & Ciências & Sudeste & USP \\
\hline 42 & 3 & Química & Sudeste & UFSCar/USP \\
\hline 43 & 3 & Biologia & Sudeste & USP \\
\hline
\end{tabular}

\section{A produção e sua distribuição de acordo com a área de pesquisa}

A Figura 2 ilustra o número de trabalhos localizados de acordo com a área de pesquisa privilegiada no enfoque dos trabalhos localizados nas revistas e apresentados nos ENPECs. Na Tabela 4 é possível observar a classificação por área de cada um dos trabalhos analisados. Vale ressaltar que a área Ciências engloba aqueles trabalhos direcionados ao ensino de Ciências, em Nível 
Fundamental, aqueles que apresentam levantamento bibliográfico sobre a argumentação no ensino de Ciências e ainda aqueles que discutem a argumentação em questões científicas gerais, mas sem especificar uma área.

Figura 2 - Trabalhos localizados nas revistas e apresentados nos ENPECs de 2001 a 2009.

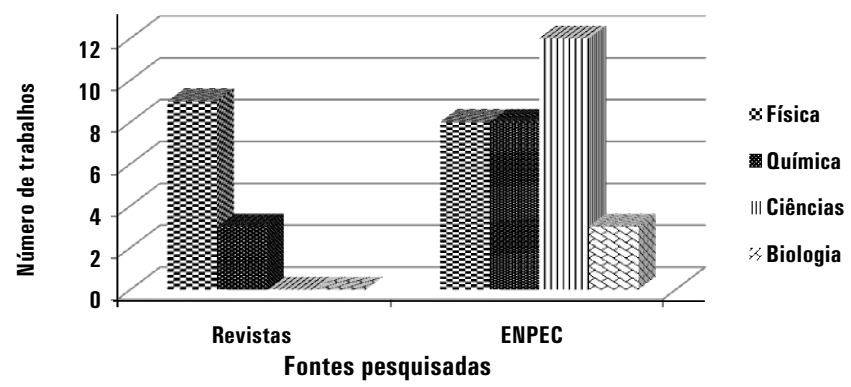

No que diz respeito à análise das revistas, nove dos doze trabalhos concernem à área de Física e três à área de Química. Nenhum trabalho relacionado ao tema com enfoque na área de Ciências ou Biologia foi localizado nas revistas direcionadas ao ensino de Ciências, nem na revista específica da área de Biologia, Revista Brasileira de Ensino de Bioquímica e Biologia Molecular. Esses números chamam a atenção para a crescente mobilização e iniciativas de publicações de artigos, por parte de pesquisadores da área de Física, em relação à prática da argumentação na sala de aula e sobre a necessidade de mais ações nessa direção nas demais áreas da Ciência.

Em relação ao ENPEC, dos 31 trabalhos localizados, oito dizem respeito à área de Física, oito à área de Química, doze à área de Ciências, e três à área de Biologia. Assim, publicações na área de Biologia são ainda pouco expressivas, se comparadas às demais áreas. Somente no último ENPEC, realizado em 2009, surgem os primeiros trabalhos concernentes a esta área.

Os resultados apontam que a área de Física tem se destacado pela quantidade de contribuições acerca do tema, principalmente pelo número de publicações em revistas da área, se comparada às áreas de Química e Biologia. Muito embora nos ENPECs o número de trabalhos pertencentes a essas áreas tenha crescido nos últimos anos, existem ainda poucos grupos que investigam o tema.

\section{A produção e sua distribuição de acordo com as regiões brasileiras}

A Figura 3 ilustra o número de trabalhos localizados nas revistas da área de Ensino de Ciências e aqueles apresentados nos ENPECs, de acordo com 
a sua distribuição por regiões geográficas do Brasil. A classificação dos trabalhos por região é apresentada na Tabela 4. É necessário destacar que na Figura 3 o número de trabalhos excede os 43 trabalhos considerados na análise, uma vez que cada trabalho apresentado por dois autores provenientes de regiões diferentes foi computado duas vezes (uma vez para cada uma das regiões).

Figura 3 - Distribuição dos trabalhos de acordo com as regiões brasileiras.

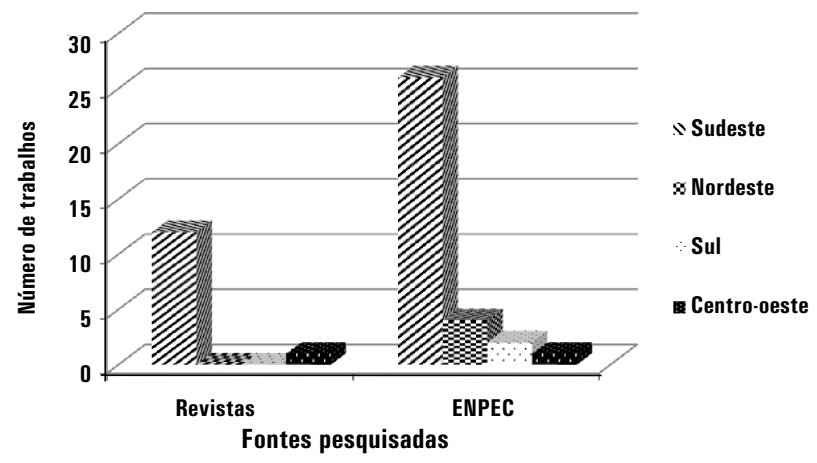

Conforme ilustra a Figura 3, a grande maioria dos trabalhos localizados nas revistas e apresentados nos eventos é proveniente da região Sudeste. O fato de a região abrigar um número elevado de Instituições de Ensino Superior (IES) de grande tradição em pesquisa no país, como a USP e a Universidade Federal de Minas Gerais (UFMG), justifica, em parte, a relevante contribuição de pesquisas na área de Ensino de Ciências. Além disso, na região Sudeste está concentrado um número elevado de programas de Pós-Graduação, outra provável razão para a maior parte das contribuições de pesquisa ser oriunda dessa região.

No que diz respeito aos trabalhos apresentados nos ENPECs, há ainda outro aspecto que pode justificar o fato de a região Sudeste apresentar a maioria dos trabalhos. Dos cinco ENPECs promovidos, três foram realizados no estado de São Paulo e dois no estado de Santa Catarina, regiões relativamente próximas. Esse favorecimento propiciado pelo local de realização dos eventos é considerável (FRANCISCO \& QUEIROZ, 2008).

A segunda região com maior produção de trabalhos sobre o tema foi a do Nordeste, porém com diferença significativa se comparada à região Sudeste. Nenhum dos trabalhos localizados nas revistas foi proveniente da região Nordeste.

$\mathrm{Na}$ região Centro-oeste foram produzidos apenas dois trabalhos sobre a temática, sendo ambos, na realidade, detentores do mesmo conteúdo, como já mencionado anteriormente, o mesmo trabalho foi publicado em fontes distintas. Essas contribuições são oriundas do Distrito Federal. No que diz respeito à 
região Sul, apenas dois trabalhos foram localizados. Esses foram apresentados no III e VI ENPEC respectivamente e, ambos, são provenientes do estado do Rio Grande do Sul. Nenhum dos trabalhos analisados é proveniente da região Norte. O isolamento típico do local e o seu distanciamento do Sudeste podem explicar, em parte, a pouca participação de pesquisadores do estado em eventos da área. No entanto, também verificamos inexistência de publicações sobre o tema nas revistas analisadas, fato que sugere a pouca atividade de pesquisa sobre o tema em universidades da região.

\section{A produção e sua distribuição de acordo com as instituições acadêmicas}

Nesse tópico apresentamos as IES às quais estão vinculados os autores dos trabalhos analisados. A Figura 4 apresenta a quantidade de trabalhos de acordo com as instituições acadêmicas às quais os mesmos são vinculados. A classificação de cada um dos trabalhos é apresentada na Tabela 4. É necessário destacar que na Figura 4 o número de trabalhos excede os 43 trabalhos considerados na análise, uma vez que cada trabalho proveniente de duas instituições diferentes foi computado duas vezes (uma vez para cada instituição).

Conforme ilustra a Figura 4, a IES que mais contribuiu com a produção de trabalhos publicados sobre argumentação nas revistas foi a UFMG. É digno de nota que, dos seis trabalhos localizados nas revistas nacionais provenientes da instituição, cinco deles (4, 7, 8, 10 e 12) são oriundos do grupo liderado por Silvania Sousa do Nascimento. A quinta contribuição da mesma instituição (2) pertence ao grupo liderado por Eduardo Fleury Mortimer. Com o mesmo número de artigos publicados nas revistas temos a USP (1, 3 e 5) e a $\operatorname{UNESP}(6,9$ e 11).

Figura 4 - Distribuição dos trabalhos de acordo com as IES.

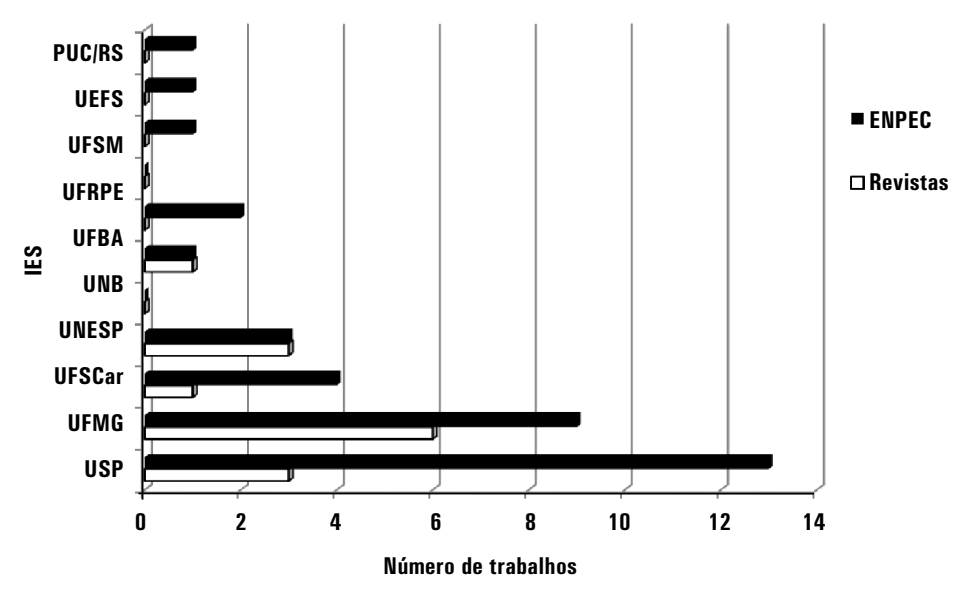


Em contraponto, no que diz respeito aos trabalhos apresentados nos ENPECs, a USP apresentou o maior número de contribuições: 13 dos 31 trabalhos apresentados nas edições do evento. O total de trabalhos é proveniente tanto de institutos pertencentes ao campus de São Paulo quanto de unidades em seus campi do interior. Destacam-se os grupos liderados por Ana Maria Pessoa de Carvalho, da Faculdade de Educação, e por Salete Linhares Queiroz, do IQSC/USP. Na região Sudeste se destacam ainda a Universidade Federal de São Carlos (UFSCar) e a Universidade Estadual Paulista (UNESP): foram apresentados quatro trabalhos provenientes da UFSCar $(25,27,39$ e 42) e três da UNESP (16, 19 e 38). Os trabalhos produzidos pela UNESP são provenientes de unidades em seus campi, como o de Bauru e o de Guaratinguetá. Dessa instituição se destaca o grupo liderado por Odete Pacubi Baierl Teixeira. Com relação à UFSCar, todos os trabalhos são provenientes de pesquisas vinculadas ao Grupo de Pesquisa em Ensino de Química do IQSC/USP.

As IES das demais regiões brasileiras apresentam contribuições em número pouco significativo nos ENPECs. Da região Sul, identificamos o trabalho 24, da Universidade Federal de Santa Maria (UFSM); e o 14, da Pontifícia Universidade Católica do Rio Grande do Sul (PUC/RS). Em relação ao Nordeste foram verificadas contribuições da Universidade Federal da Bahia (UFBA) e da Universidade Estadual de Feira de Santana (UEFS) (23 e 32); e da região Centro-oeste, o trabalho 13, da Universidade de Brasília (UnB).

\section{A produção e sua distribuição de acordo com os níveis de escolaridade}

A identificação do nível escolar ao qual se relacionam os trabalhos foi realizada por meio da utilização dos descritores sobre níveis escolares estabelecidos por Megid Neto (1999). Alguns dos elementos considerados para a identificação do nível escolar foram: os sujeitos participantes na pesquisa, os materiais didáticos avaliados, os programas de ensino propostos e as experiências educacionais relatadas na pesquisa. A Tabela 5 apresenta a classificação dos trabalhos de acordo com o nível de escolaridade e foco temático. Aqueles trabalhos classificados no foco temático Estratégias promotoras da argumentação são ainda classificados de acordo com o tipo de abordagem empregada na pesquisa (científica ou sócio-científica). Tais aspectos serão discutidos no próximo tópico. 
Tabela 5 - Classificação dos trabalhos de acordo com nível de escolaridade e foco temático, onde $\mathrm{F}=$ Fundamental, $\mathrm{M}=$ Médio, $\mathrm{S}=$ Superior, $\mathrm{G}=$ Geral.

\begin{tabular}{|c|c|c|c|}
\hline$N^{0}$ doTrabalho & Tabela & Nível & Foco temético/Abordagem \\
\hline 1 & 2 & $\mathbf{F}$ & Estratégias promotoras da argumentação/científica \\
\hline 2 & 2 & M & $\begin{array}{l}\text { Estratégias promotoras da argumentação/sócio-científica } \\
\text { Formação de professores }\end{array}$ \\
\hline 3 & 2 & M & $\begin{array}{c}\text { Estratégias promotoras da argumentação/científica } \\
\text { Formação de professores }\end{array}$ \\
\hline 4 & 2 & M & Estratégias promotoras da argumentação/científica \\
\hline 5 & 2 & $\mathbf{S}$ & Estratégias promotoras da argumentação/sócio-científica \\
\hline 6 & 2 & M & Estratégias promotoras da argumentação/científica \\
\hline 7 & 2 & $\mathbf{S}$ & Formação de professores \\
\hline 8 & 2 & $\mathbf{S}$ & Formação de professores \\
\hline 9 & 2 & M & Estratégias promotoras da argumentação/científica \\
\hline 10 & 2 & $\mathbf{S}$ & Formação de professores \\
\hline 11 & 2 & M & Estratégias promotoras da argumentação/científica \\
\hline 12 & 2 & $\mathbf{S}$ & Formação de professores \\
\hline 13 & 3 & M & $\begin{array}{l}\text { Estratégias promotoras da argumentação/sócio-científica } \\
\text { Formação de professores }\end{array}$ \\
\hline 14 & 3 & $\mathbf{S}$ & $\begin{array}{c}\text { Estratégias promotoras da argumentação/científica } \\
\text { Formação de professores }\end{array}$ \\
\hline 15 & 3 & $\mathbf{F}$ & Estratégias promotoras da argumentação/científica \\
\hline 16 & 3 & $\mathbf{F}$ & $\begin{array}{c}\text { Estratégias promotoras da argumentação/científica } \\
\text { Formação de professores }\end{array}$ \\
\hline 17 & 3 & $\mathbf{S}$ & Formação de professores \\
\hline 18 & 3 & $\mathbf{S}$ & Formação de professores \\
\hline 19 & 3 & M & $\begin{array}{l}\text { Estratégias promotoras da argumentação/científica } \\
\text { Formação de professores }\end{array}$ \\
\hline 20 & 3 & $\mathbf{F}$ & Espaço para argumentação \\
\hline 21 & 3 & $\mathbf{G}$ & Levantamento bibliográfico \\
\hline 22 & 3 & $\mathbf{S}$ & Formação de professores \\
\hline 23 & 3 & $\mathbf{S}$ & Estratégias promotoras da argumentação/científica \\
\hline 24 & 3 & G & Levantamento bibliográfico \\
\hline 25 & 3 & $\mathbf{S}$ & Espaço para argumentação \\
\hline 26 & 3 & M & Estratégias promotoras da argumentação/científica \\
\hline 27 & 3 & $\mathbf{S}$ & Estratégias promotoras da argumentação/científica \\
\hline 28 & 3 & M & Estratégias promotoras da argumentação/científica \\
\hline 29 & 3 & M & Elaboração de modelo \\
\hline 30 & 3 & M & Elaboração de modelo \\
\hline 31 & 3 & M & Estratégias promotoras da argumentação/científica \\
\hline 32 & 3 & M & Estratégias promotoras da argumentação/científica \\
\hline 33 & 3 & $\mathbf{S}$ & Formação de professores \\
\hline 34 & 3 & M & Análise de material didático \\
\hline 35 & 33 & G & Levantamento bibliográfico \\
\hline 36 & 3 & M & Estratégias promotoras da argumentação/científica \\
\hline 37 & 3 & $\mathbf{S}$ & Estratégias promotoras da argumentação/científica \\
\hline 38 & 3 & M & Estratégias promotoras da argumentação/sócio-científica \\
\hline 39 & 3 & $\mathbf{S}$ & Mecanismos de ensino da argumentação \\
\hline 40 & 3 & M & Estratégias promotoras da argumentação/sócio-científica \\
\hline 41 & 3 & $\mathbf{F}$ & Estratégias promotoras da argumentação/científica \\
\hline 42 & 3 & $\mathbf{S}$ & Elaboração de modelo \\
\hline 43 & 3 & M & Estratégias promotoras da argumentação/científica \\
\hline
\end{tabular}

A Figura 5 ilustra a distribuição de trabalhos de acordo com o nível de escolaridade. Cabe destacar que foram classificados na categoria Geral três trabalhos que apresentam levantamento bibliográfico sobre a temática (21, 24 e 35). 
Figura 5 - Distribuição dos trabalhos de acordo com o nível de escolaridade.

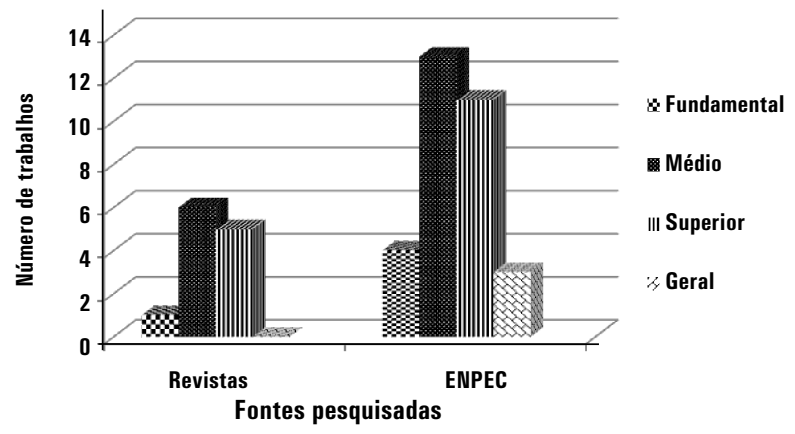

Conforme verificamos na Figura 5, o nível de escolaridade que mais se destacou nos trabalhos analisados foi o Ensino Médio. Observamos que a maioria dos trabalhos direcionados a esse nível de ensino apresenta como objetivo principal o desenvolvimento da capacidade de argumentação dos estudantes, por meio da promoção de estratégias de ensino com abordagem em questões científicas ou sócio-científicas. Esse propósito apontado na maioria dos trabalhos encontra respaldo na colocação de Cachapuz et al (2005), quando afirmam que o objetivo da escola básica não é formar futuros cientistas, mas permitir que os alunos possam entender o mundo discutindo e compreendendo os fenômenos científicos e tecnológicos.

Trabalhos direcionados ao Ensino Superior também aparecem em frequência significativa. Dentre os grupos que trabalham com a temática no Ensino Superior se destacam o grupo liderado por Silvania Souza do Nascimento, cujos trabalhos têm como foco principal a formação inicial de professores de Física; e o grupo liderado por Salete Linhares Queiroz, que apresenta estudos voltados ao aprimoramento de habilidades argumentativas de graduandos em Química.

O nível de escolaridade menos privilegiado nos trabalhos foi o Nível Fundamental. Nesse nível de ensino se destacam os trabalhos desenvolvidos pelo grupo de Ana Maria Pessoa de Carvalho, cujo enfoque se dá no desenvolvimento de habilidades argumentativas em aulas de conhecimento físico com crianças.

\section{A produção e sua distribuição de acordo com o foco temático}

Com base na análise das produções nacionais supracitadas, constatamos que as pesquisas nelas reportadas se relacionam, principalmente, aos seguintes focos temáticos: (a) Estratégias promotoras da argumentação: trabalhos que apresentam estratégias de ensino que visam à promoção da argumentação com abordagem em aspectos científicos e/ou sócio-científicos; (b) Formação de professores: 
trabalhos que apresentam discussões acerca do papel do professor na promoção da argumentação na sala de aula, em contextos de formação inicial ou continuada; (c) Elaboração de modelos: trabalhos que apresentam modelos que visam avaliar argumentos produzidos pelos alunos mediante distintas situações apresentadas em ambientes de ensino-aprendizagem de Ciências; (d) Espaço para a argumentação: trabalhos que apresentam questões relacionadas ao espaço destinado à prática da argumentação em ambientes de ensino-aprendizagem de Ciências; (e) Mecanismos de ensino da argumentação: trabalhos que discutem sobre a viabilidade do emprego de mecanismos que objetivam o ensino da argumentação aos estudantes. $\mathrm{O}$ mesmo se diferencia do foco Estratégias promotoras da argumentação, pelo fato de que, no primeiro, os alunos não são instruídos em relação aos componentes presentes na argumentação; (f) Levantamento bibliográfico: trabalhos que apresentam levantamento bibliográfico sobre pesquisas que tratam de questões concernentes à argumentação no ensino de Ciências e (g) Análise de material didático: trabalhos que analisam aspectos da argumentação existente em materiais didáticos.

A Figura 6 apresenta a distribuição dos trabalhos de acordo com seu foco temático. Cabe ressaltar que os trabalhos 2, 3, 13, 14, 16 e 19 foram classificados em dois focos temáticos: estratégias promotoras da argumentação e formação de professores. Assim, o número verificado na Figura 6 ultrapassa o total de 43 trabalhos.

A seguir apresentamos uma breve discussão a respeito dos trabalhos relacionados a cada um dos focos temáticos, de acordo com a Figura 6. Devido a limitações de espaço, que são determinadas pela Revista Ensaio, discutimos de forma particular apenas alguns trabalhos que julgamos representar de forma mais ilustrativa a natureza dos estudos classificados em cada um dos focos.

Estratégias promotoras da argumentação: dos 43 trabalhos localizados, 25 discutem questões relacionadas ao emprego de estratégias promotoras da argumentação. Classificamos ainda essas pesquisas de acordo com o tipo de abordagem envolvida no estudo, ou seja, verificamos se as estratégias empregadas envolviam questões de natureza científica ou sócio-científica.

Figura 6 - Distribuição dos trabalhos de acordo com o foco temático.

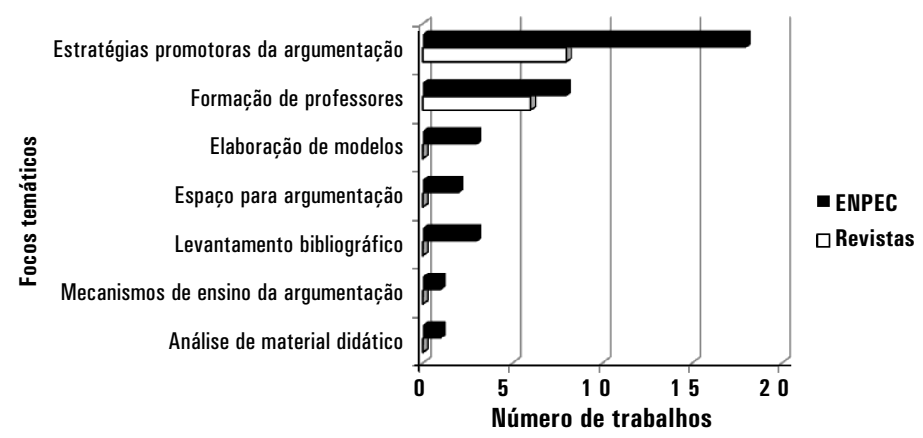


Oito dos trabalhos localizados nas revistas apresentam esse foco temático, seis com abordagem em aspectos científicos e dois com abordagem em aspectos sócio-científicos. No que diz respeito à primeira abordagem, o trabalho 1 (Tabela 2) foi desenvolvido com o intuito de verificar em que medida as atividades propostas estimularam a elaboração de argumentos por crianças, no Ensino Fundamental, e analisar a qualidade das argumentações em discussões sobre assuntos científicos, no caso, sobre a observação da variação do alcance de um jato em função da altura de uma coluna de água. Segundo as autoras, o espaço concedido à argumentação nesse trabalho revelou-se como uma oportunidade para o incentivo à cooperação entre os alunos, incluindo ao mesmo tempo o respeito às diferentes explicações e a autoconfiança para posicionamentos contrários aos dos colegas.

No que diz respeito à abordagem sócio-científica, no trabalho 2 (Tabela 2) o debate foi utilizado como estratégia para introduzir questões sóciocientíficas na sala de aula. O estudo foi desenvolvido numa escola do Ensino Médio e o debate promovido tratava da tríade "Ciência, Religião e Magia", com o principal propósito de desenvolver a argumentação dos estudantes e discutir a natureza do conhecimento científico. Segundo os autores, a intenção era que o aluno fosse capaz de apresentar justificativas que sustentassem o seu posicionamento sobre o tema.

Formação de professores: verificamos a existência de 14 trabalhos com esse enfoque. Nas revistas analisadas foram localizados seis deles, enquanto os demais foram apresentados nos ENPECs. No trabalho 7 (Tabela 2), por exemplo, os autores apresentam considerações a respeito de contribuições aos processos de ensino-aprendizagem na formação inicial de professores de Física à luz de referenciais teóricos do campo da argumentação. Para tanto, selecionaram e analisaram, segundo esses referenciais, um episódio de ensino com contraposição de ideias sobre a noção de repouso mecânico. Nesse processo, averiguaram como a ação pedagógica do professor formador refletiu-se na produção de argumentos por parte dos licenciandos. Ao contrastar os resultados obtidos com aqueles observados em pesquisas sobre argumentação na Educação Básica, os autores observaram a não ocorrência de intervenções eliciativas por parte do formador. As interações argumentativas seguiram seu curso a partir da contraposição de ideias iniciais fornecida pelo mesmo, mas uma vez iniciado, o processo argumentativo contou com uma dinâmica que não necessitou de feedbacks para se manter, embora o formador fizesse algumas intervenções avaliativas.

Elaboração de modelos: identificamos três trabalhos com esse foco, todos relacionados à área de Química e apresentados nos ENPECs. Por exemplo, no trabalho 29 (Tabela 3) os autores elaboraram um esquema de análise que representa o relacionamento entre os componentes do argumento, contra-argumento e refutação presentes no raciocínio argumentativo. Assim, identificaram as principais habilidades argumentativas que podem ser desenvolvidas no ensino de Ciências e discutiram algumas questões presentes no instrumento proposto que 
permitem acessá-las. No mesmo evento, o trabalho 30 descreve como o esquema de análise foi validado.

Espaço para a argumentação: identificamos dois trabalhos com esse foco temático, ambos apresentados nos ENPECs. No trabalho 25 ('Tabela 3), por exemplo, os autores buscaram determinar se professores de ensino Superior de Química ofereciam oportunidades aos estudantes para o desenvolvimento de habilidades argumentativas. Para tanto, aulas de diferentes disciplinas, de caráter teórico e experimental, distribuídas em um curso de Bacharelado em Química foram observadas. Foi verificada a predominância da fala dos professores nas aulas, o que não favorecia a ocorrência de discussões sobre questões científicas.

Mecanismos de ensino da argumentação: identificamos um único trabalho com esse foco temático, apresentado no VII ENPEC. O trabalho 39 (Tabela 3) teve como objetivo investigar sobre a efetividade do ensino da estrutura de um "bom argumento" como apoio ao desenvolvimento de habilidades argumentativas de alunos de um curso de Bacharelado em Química. Para tanto foi realizada uma análise comparativa da argumentação dos alunos quando submetidos a diferentes contextos de ensino: no primeiro a argumentação foi totalmente espontânea e nenhuma orientação no sentido de como argumentar foi oferecida; no segundo, uma série de atividades desenvolvidas com o intuito de torná-los conhecedores dos elementos que constituem um "bom argumento" foi realizada. Os resultados não apresentaram diferença significativa entre a qualidade dos argumentos produzidos nos dois contextos de ensino.

Levantamento bibliográfico: identificamos três trabalhos com esse foco temático, todos apresentados no VII ENPEC. No trabalho 24 (Tabela 3), os autores visaram compreender como estavam sendo desenvolvidas, em aulas de Ciências, atividades que buscavam, além de explorar o conhecimento científico, estimular os alunos a desenvolver uma argumentação consistente. Dentre outros aspectos, perceberam grande preocupação na compreensão da linguagem utilizada por professores de modo a estimular os alunos a expressar e a defender seus pontos de vista. Os autores ressaltam que ao mesmo tempo em que professores buscam desenvolver a capacidade argumentativa dos alunos, eles, em momentos coletivos de discussão, demonstram falta de uma melhor preparação quanto ao tipo de discurso a ser utilizado e quanto à maneira de conduzir as discussões.

Análise de material didático: identificamos um único trabalho com esse foco temático, apresentado no VII ENPEC. No trabalho 34 (Tabela 3) os autores buscaram identificar a presença de funções didáticas, como a argumentação, na sequência didática de um livro de Química. Os resultados obtidos apontam para a predominância de aplicações e definições no material analisado, em detrimento da argumentação, explicação e problematização. 


\section{CONSIDERAÇÕES FINAIS}

O estudo revela que, a partir de 2007, foi atribuída ao assunto considerável importância, o que é corroborado pelo aumento no número de trabalhos desde então. No entanto, a maior parte deles foi apresentada nos ENPECs, o que sugere que vários grupos começaram a investigar a temática recentemente, podendo se estimar a consolidação dessa linha de pesquisa nos referidos grupos nos próximos anos, com a posterior publicação de trabalhos também em revistas "qualizadas".

Investigações concernentes à área de Física são reportadas com mais constância na literatura se comparada às demais áreas, enquanto o nível de escolaridade privilegiado nos estudos é o Ensino Médio. Nessa perspectiva, o estudo aponta para a necessidade de realização de mais trabalhos nas áreas de Química e Biologia, assim como de pesquisas que abarquem todos os níveis de ensino.

No que diz respeito às regiões geográficas de produção dos trabalhos, evidenciamos maior participação de pesquisadores da região Sudeste. Com exceção de uma única publicação em revista, as demais são todas provenientes dessa região (Figura 3), o que sugere uma concentração de pesquisadores que investigam o assunto a mais tempo nessa região. Assim, o estabelecimento de parceiras entre tais pesquisadores e os das outras regiões é recomendável para que as discussões sobre o assunto progridam no nosso país.

Em relação aos focos temáticos, percebemos que a maioria das pesquisas ainda se concentra principalmente na formação de professores e no desenvolvimento de estratégias promotoras da argumentação. Estes são, inclusive, os únicos focos tratados nas publicações presentes nas revistas (Figura 6). Pesquisas que tratam, por exemplo, da elaboração de modelos para análise da argumentação e de mecanismos de ensino da argumentação são escassas, sugerindo a existência de assuntos e questionamentos que precisam ser mais amplamente investigados no contexto nacional.

Por fim, cabe ainda destacar que, muito embora sua inserção no currículo seja bastante recomendada por pesquisadores da área de ensino de Ciências (KOLSTO, 2006; SADLER \& DONNELLY, 2006; BRAUND et al, 2007; ZEIDLER et al, 2009), apenas cinco dos trabalhos analisados apresentam estratégias com abordagem em questões de natureza sócio-científica. Em contraponto, na literatura internacional são abundantes as pesquisas que abordam questões dessa natureza. 


\section{REFERÊNCIAS BIBLIOGRÁFICAS}

BRAUND, M. et al. Comparing the effect of scientific and socio-scientific argumentation tasks: lessons from South Africa. School Science Review, v. 88, n. 324, p. 67-76, 2007.

CACHAPUZ, A. et al. Necessária renovação do ensino de Ciências. São Paulo: Cortez, 2005.

ERDURAN, S.; JIMÉNEZ-ALEIXANDRE, M. P. Argumentation in Science Education: Perspectives from Classroom-Based Research. Dordrecht: Springer, 2008.

FRANCISCO, C. A; QUEIROZ, S. L. A produção sobre o ensino de Química nas Reuniões Anuais da Sociedade Brasileira de Química: uma revisão. Química Nova, v. 31, n. 8, p. 2100-2110, 2008.

KOLSTO, S. D. Patterns in student's argumentation confronted with a risk-focused socio-scientific issue. International Journal of Science Education, v. 28, n. 14, p. 1689-1716, 2006.

LEE, M.; WU, Y.; TSAI, C. Research Trends in Science Education from 2003 to 2007: A content analysis of publications in selected journals. International Journal of Science Education, v. 3 1, n. 15, p. 1999-2020, 2009.

MARTINS, I. Argumentación en Textos de una Revista de Educación de Profesores. Un Ejercicio de Análisis Basado en Conceptos Bakhtinianos de Gênero y Lenguaje Social. Educación Química, v. 20, n. 2, p. 126-136, 2009.

MEGID NETO, J. Tendências da pesquisa acadêmica sobre o ensino de Ciências no Nível Fundamental. 1999.236 f. Tese (Doutorado) - Faculdade de Educação, Universidade de Campinas (UNICAMP), Campinas, 1999.

NASCIMENTO, S. S.; PLANTIN, S. (org). Argumentação e ensino de Ciências. Curitiba: CRV, 2009. NASCIMENTO, S. S; VIEIRA, R. D. Contribuições e limites do padrão de argumento de Toulmin aplicado em situações argumentativas de sala de aula de Ciências. Revista Brasileira de Pesquisa em Educação em Ciências, v. 8, n. 2, 2008.

QUEIROZ, S. L.; SÁ, L. P. O espaço para a argumentação no Ensino Superior de Química. Educación Química, v. 20, n. 2, p. 104-110, 2009.

SADLER, T. D. Promoting discourse and argumentation in science teacher education. Journal of Research in Science Teaching, v. 17, n. 4, p. 323-346, 2006.

SADLER, T. D.; DONNELLY, L. A. Socioscientific argumentation: the effects of content knowledge and morality. International Journal of Science Education, v. 28, n. 12, p. 1463-1488, 2006.

SANTOS, W. P.; MORTIMER, E. F.; SCOTT, P. H. A argumentação em discussões sócio-científicas: reflexões a partir de um estudo de caso. Revista Brasileira de Pesquisa em Educação em Ciências, v. 1, n. 1, p. 140-152, 2001.

SCHNETZLER, R. P. A pesquisa em ensino de Química no Brasil: conquistas e perspectivas. Quimica Nova, v. 25, suplemento 1, p. 14- 24, 2002.

ZEIDLER, D. L.; SADLER, T. D.; SCOTT, A.; CALLAHAN, B. E. Advancing reflective judgment through socioscientific issues. Journal of Research in Science Teaching, v. 46, n. 1, p. 74-101, 2009.

Data de recebimento: $17 / 07 / 2010$

Data de aprovação: 28/10/2010

Data da versão final: 01/12/2010 